\title{
Torta de cupuaçu (Theobroma grandiflorum) em dietas para juvenis de tambaqui (Colossoma macropomum cuvier, 1818)
}

\author{
Débora Tatyane Oliveira Xavier ${ }^{1 *}$, Verucia Maria Dias Brandão², Fabricio Nilo da Silva ${ }^{3}$, \\ Lian Valente Brandão ${ }^{4}$, Raimundo Aderson Lobão de Souza 5 .
}

\begin{abstract}
${ }^{I}$ Tecnóloga em Aquicultura, Especialista em Gestão, Consultoria, Auditoria, Perícia e Fiscalização Ambiental pelo Instituto de Estudos Superiores da Amazônia (IESAM/PA), Mestranda do Programa de Pós-Graduação em Aquicultura e Recursos Aquáticos Tropicais da Universidade Federal Rural da Amazônia (UFRA). Av. Perimetral, 2501-Universitário - Belém - PA. E-mail: debyatm@hotmail.com

${ }^{2}$ Médica Veterinária pela Universidade Federal do Pará (UFPA Campus Castanhal). Av. dos Universitários Jaderlândia, Castanhal-PA.E-mail: veruciadias@yahoo.com.br

${ }^{3}$ Doutorando do Programa de Pós-Graduação em Ciência Animal na área de concentração Ecologia Aquática e Aquicultura pela (Universidade Federal do Pará - UFPA). Professor do quadro efetivo do Ensino Básico, Técnico e Tecnológico no Instituto Federal de Educação, Ciência e Tecnologia do Pará - Campus Breves. E-mail: fabricio_nilo@hotmail.com

${ }^{4}$ Doutor em Biologia de Água Doce e Pesca Interior, Coordenador Geral de Ensino de Graduação do Instituto Federal de Educação, Ciência e Tecnologia do Pará (IFPA Campus Castanhal). Núcleo de Pesquisa Aplicada em Pesca e Aquicultura Norte 03. Rod. BR 316, Km, 62 - Castanhal - PA, Bairro: Saudade. E-mail: lianpesca@ yahoo.com.br

${ }^{5}$ Doutor em Ecologia e Recursos Naturais, Docente do programa de pós-graduação em aquicultura e recursos aquáticos tropicais (UFRA). Av. Perimetral, 2501-Universitário-Belém - PA. E-mail: adersonlobao@globo.com

*Autor para correspondência.
\end{abstract}

RESUMO. Esta pesquisa foi realizada com o objetivo de avaliar o ganho de peso do tambaqui (Colossoma macropomum) utilizando dietas contendo níveis crescentes de torta de cupuaçu $(0 \%, 10 \%, 20 \%$ e $30 \%)$. O experimento foi realizado na piscicultura São Pedro, Santa Luzia do Pará - PA. Para avaliar o ganho de peso foram utilizados 240 juvenis de tambaqui, com 100g inicial. Foram utilizadas 12 unidades experimentais com tamanho de $1,7 \mathrm{x} 2,55 \mathrm{~m}^{2}$ onde cada unidade alocava 20 peixes, sendo os teores separados em triplicatas, em um período de 60 dias. $O$ resultado comprovou que os peixes alimentados com a ração sem inclusão da torta de cupuaçu (ração controle), obteve o melhor desempenho que os peixes alimentados com os demais níveis $(10 \%, 20 \%$ e $30 \%$ respectivamente). Concluindo que o aumento da inclusão interfere de forma negativa no desempenho zootécnico da espécie.

Palavras chave: Desempenho, experimento, inclusão, ração

\section{Cupuaçu (Theobroma grandiflorum) pie in diet for tambaqui (Colossoma macropomum Cuvier, 1818)}

\begin{abstract}
This research was conducted to evaluate the weight gain tambaqui (Colossoma macropomum) using diets containing increasing levels of pie cupuaçu $(0 \%$, $10 \%, 20$ and 30\%). The experiment was conducted at São Pedro fish farming, Santa Luzia Para-PA. To assess weight gain were conducted with 240 tambaqui, starting with $100 \mathrm{~g}$. A total of 12 experimental units with size of $1.7 \times 2.55 \mathrm{~m} 2$ where each unit alocava 20 fish, with levels separated in triplicate, in a period of 60 days. The result showed that the fish fed the diet without inclusion of pie cupuaçu (control diet), had the best performance that fish fed the other levels $(10 \%, 20 \%$ and 30\% respectively). In conclusion that increasing the inclusion interferes negatively on the performance of the species.
\end{abstract}

Keywords: Performance, experiment, inclusion, feed

\section{Introdução}

A aquicultura é a atividade agropecuária em maior expansão mundial (Pianesso et al., 2013). O cultivo de organismos aquáticos, cujo ciclo de vida em condições naturais, ocorre total ou parcialmente em meio aquático. Enquanto 
atividade econômica está em plena expansão em todo o mundo com um crescimento médio anual de 8,3\% (1970-2008) (FAO, 2010). O cultivo de organismos aquáticos destaca-se como o setor de produção animal que mais cresce no mundo (DeCarvalho et al., 2013). A Organização das Nações Unidas para Alimentação e Agricultura (FAO) destaca o Brasil como um dos maiores produtores de pescado do mundo, com uma estimativa de 20 milhões de toneladas em 2030 (FAO, 2015)).

Na região norte, com destaque para o estado de Rondônia, o tambaqui tem despontado como a principal espécie de peixe nativa cultivada (Melo et al., 2001)). No estado do Pará, a piscicultura é a atividade aquícola que mais se destaca, está distribuída em praticamente todos os municípios, essa atividade exibe uma diversidade de produtores desde o cultivo estritamente de subsistência ao grande produtor voltado para a exportação (De-Carvalho et al., 2013).

O crescimento positivo na produção do tambaqui está diretamente relacionado à dieta empregada no cultivo da espécie (Bezerra et al., 2014). O sucesso de sua criação em cativeiro se deve aos satisfatórios índices zootécnicos, rusticidade no manejo, ser de fácil reprodução em ambientes de confinamento, proporcionar uma continua oferta de alevinos e ter grande aceitabilidade no mercado consumidor (Sandoval $\mathrm{Jr}$ et al., 2013). O tambaqui (Colossoma macropomum) é a espécie que vem sendo amplamente cultivada, apresenta excelente desempenho zootécnico e adaptação aos diferentes sistemas de criação, alto valor comercial, excelente aceitação pelo consumidor, hábito alimentar onívoro/frugívoro/ zooplanctôfago e pode ser cultivado em altas densidades (Villacorta-Correa, 1997, Melo et al., 2001, Claro-Jr et al., 2004).

As condições do solo da Amazônia, de forma geral, não propiciam a produção em massa de grandes plantações de grãos. Desta forma, a disponibilidade regional de ingredientes convencionais para a formulação de rações é escassa ou inexistente (Dairiki \& Silva, 2011). Desta forma, muitas dessas fontes são "importadas" de outras regiões, com alto custo de transporte, o que onera a produção de rações e, por fim, o custo de produção dos peixes (Nwannaa et al., 2008). Isto faz com que o gasto com dietas para peixes representem entre 60 a $70 \%$ dos custos da produção (Rotta, 2002). Deste modo, os experimentos relacionados à utilização de rações devem levar em consideração o desempenho produtivo (Chagas et al., 2005) e, principalmente, o rendimento de carcaça, que compensem os custos com alimentação. Segundo Baldisserotto (2004), os ingredientes usados na elaboração de um alimento balanceado para peixes são variados e sua inclusão nas fórmulas das rações depende da sua disponibilidade na região e seu custo.

Com relação a esta disponibilidade, o Brasil se destaca como sendo um dos maiores produtores mundial de frutas que é destinado basicamente ao consumo in natura, mas que se deterioram rapidamente (Santos et al., 2008)). Dentre estas frutas, sobressai o cupuaçu, uma fruta em forma de cilindros com extremidades arredondadas que apresentam características físicas e químicas bastantes variáveis. Possui excelente potencial de mercado em razão de suas características e do aroma agradável que exalam (Calzavara et al., 1984, Souza et al., 1996).

O cupuaçu (Theobroma grandiflorum) é rico em proteínas, cálcio, fósforo e vitaminas A, B1, B2 e C. Além das vitaminas e sais minerais, a fruta é rica em pectina, uma fibra solúvel que ajuda a manter bons níveis de colesterol. Sua casca é bastante dura, é utilizada como adubo orgânico(Carvalho et al., 2005, Vieira et al., 2000). A torta do cupuaçu é o resíduo da extração do óleo da semente seca, livre de resíduo da polpa, por prensagem mecânica, através do qual se retira cerca de $80 \%$ do óleo total da semente, resultando um resíduo com aproximadamente $11 \%$ de extrato etéreo. Esses resíduos possuem um alto teor de fibra bruta (Carvalho et al., 2005, Sousa et al., 2011).

O excesso de fibra na dieta diminui a digestibilidade dos nutrientes e aumenta a produção de resíduo fecal, contribuindo para a poluição do ambiente aquático (NRC, 2011). Neste sentido, faz-se necessário a realização de estudos sobre alternativas para a melhoria das dietas visando o maior aproveitamento pelos peixes, utilizando ingredientes regionais com potencial para utilização em rações comerciais.

Diante do exposto, com o objetivo de contribuir com uma proposta maior sobre o uso de ingredientes alternativos, o presente trabalho avaliou o efeito da substituição parcial da ração com a torta de cupuaçu sobre o ganho de peso de juvenis de tambaqui (Colossoma macropomum Cuvier, 1818). 


\section{Material e Métodos}

A pesquisa foi conduzida na Piscicultura São Pedro, localizada em Santa Luzia, no estado do Pará, pertecente a microrregião de Guamá e mesorregião do Nordeste Paraense (Figura 1).

O experimento teve a duração de 60 dias de cultivo. Para a realização desta pesquisa foi utilizado um viveiro escavado de $10,20 \mathrm{~m}$ de comprimento e $4,11 \mathrm{~m}$ de largura, este espaço foi dividido em 12 unidades experimentais de $2,55 \mathrm{~m}$ x $1,7 \mathrm{~m}$, correspondendo $4,33 \mathrm{~m}^{2}$ para cada tratamento. Primeiramente foi realizada uma calagem inicial para desinfecção de organismos indesejáveis e logo após 07 dias uma adubação com esterco de gado para o aumento da produtividade primária nos viveiros, disposto na (Figuras 2 - 4).
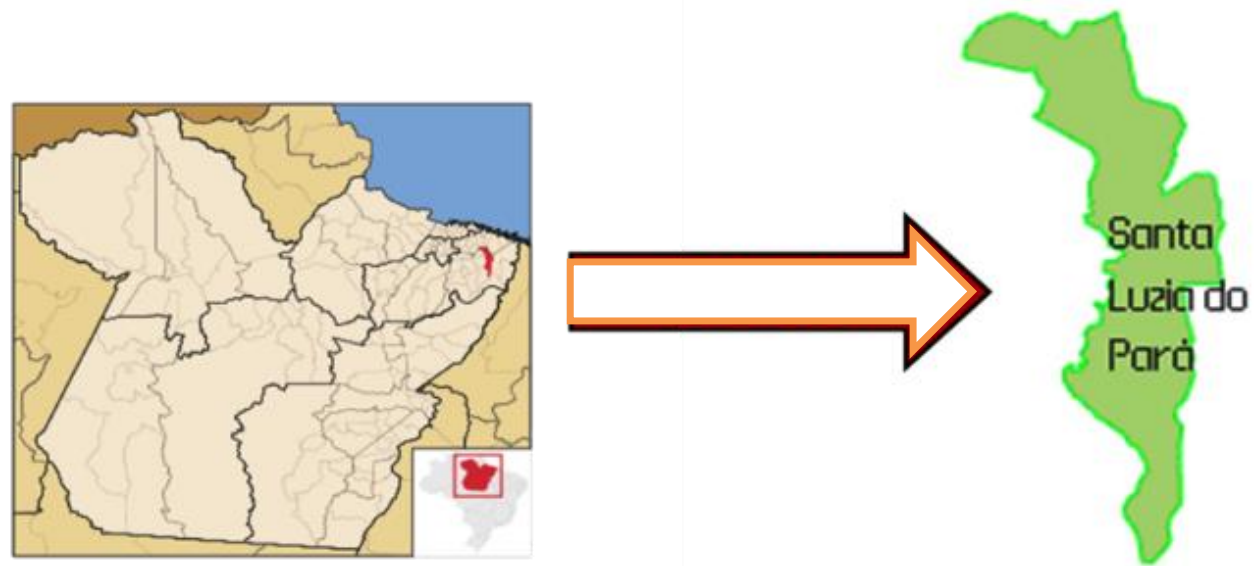

Figura 1. Mapa da Região Metropolitana de Belém, indicando a Cidade de Santa Luzia do Pará. Fonte: santaluziapontocom.blogspot.com.br.

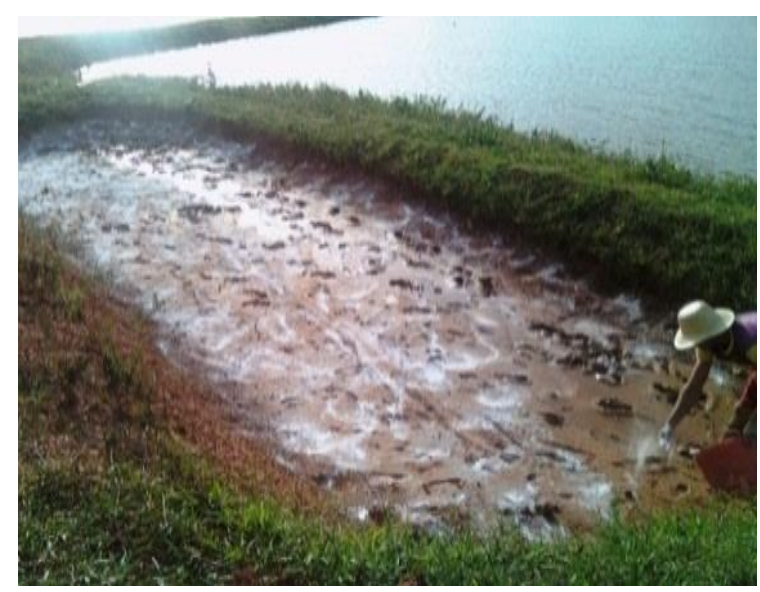

Figura 2. Calagem e adubação do viveiro.

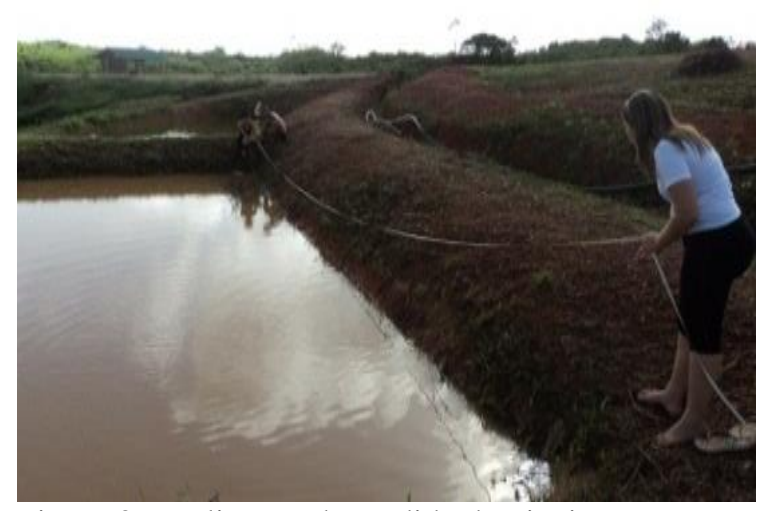

Figura 3. Realização da medida do viveiro.

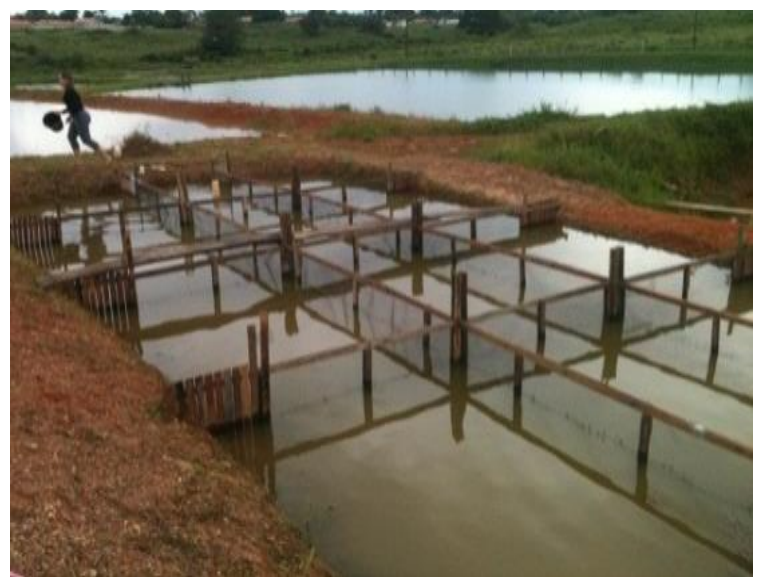

Figura 4 Divisão do viveiro em 12 unidades experimentais.

Inicialmente foram adquiridos dois milheiros de alevinos de C. macropomum. Estes animais permaneceram confinados em um tanque-rede por 30 dias sendo alimentados com uma ração comercial de $50 \%$ (proteína bruta) PB até atingirem um peso médio de 100 gramas. Após a biometria inicial, foram selecionados para pesquisa 240 espécimes com peso inicial uniforme. $\mathrm{O}$ delineamento experimental foi do tipo inteiramente casualizado, apresentando 4 tratamentos com 3 repetições, onde cada unidade experimental alocava 20 peixes (Figura 5). 

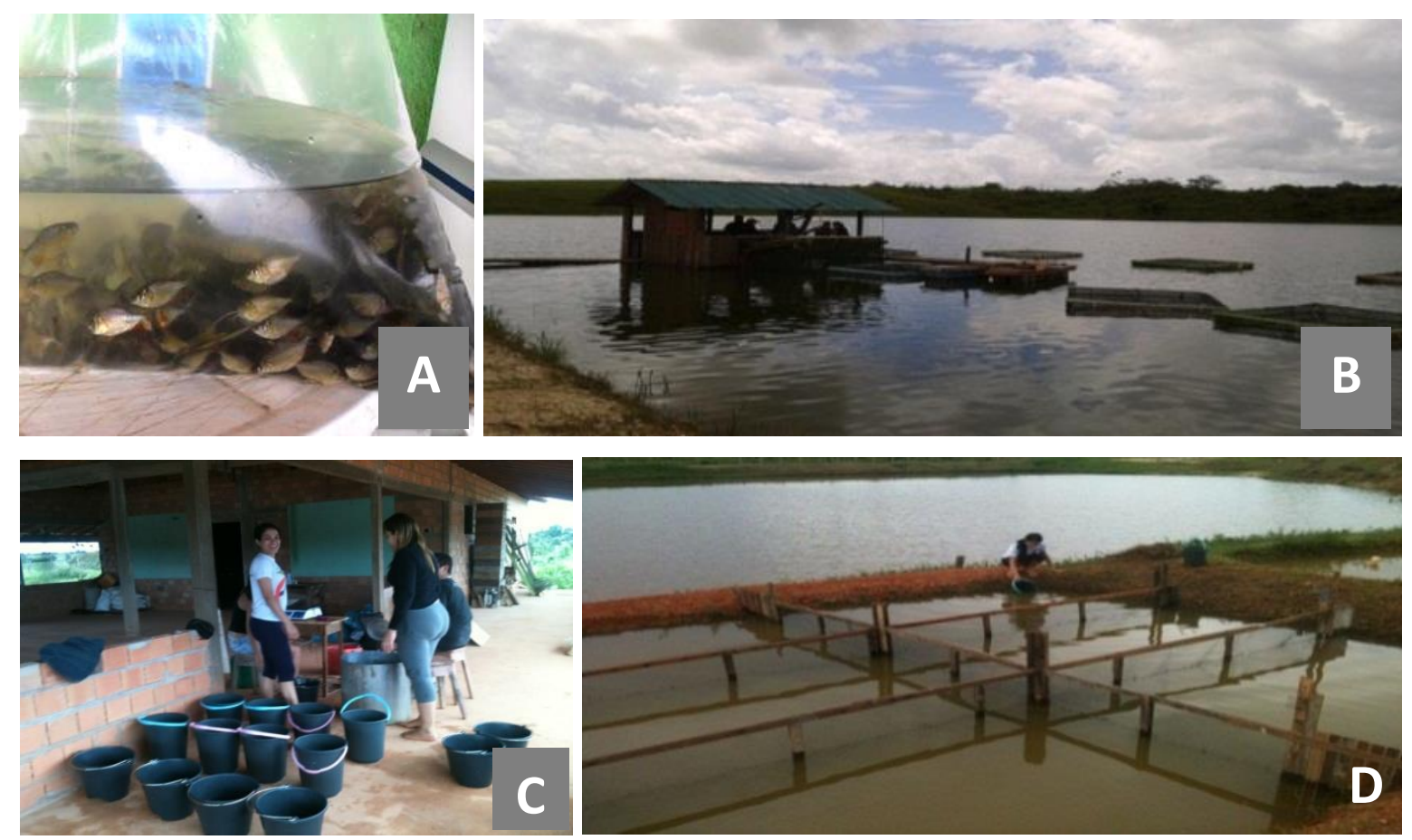

Figura 5. A. Alevinos; B. Confinamento dos alevinos em tanque-rede até atingirem um peso médio de $100 \mathrm{~g}$; C. Realização da biometria; D. Transferência dos peixes para as unidades experimentais.

Os tratamentos foram constituídos por uma dieta referência a base ingredientes de origem vegetal, como farelo de soja, farelo de trigo, farelo de milho, premix, óleo de soja, formuladas de modo a conterem níveis de inclusão de torta de cupuaçu $(10 \%, 20 \%, 30 \%)$ e uma ração controle sem adição do ingrediente alternativo (Tabela 1) e (Figura 6).
Após a pesagem conforme os teores de inclusão, os ingredientes foram misturados em um recipiente. Posteriormente a essa homogeneização, o material foi pelletizado em uma máquina moedora, formando pellets de $2 \mathrm{~mm}$ de diâmetro. Estas dietas elaboradas foram expostas ao sol durante 6 horas antes de armazenagem, para evitar o aparecimento de fungos.

Tabela 1. Composição centesimal dos ingredientes das rações usadas no teste de desempenho produtivo de juvenis de tambaqui alimentados com rações peletizadas contendo níveis crescentes de torta de cupuaçu.

\begin{tabular}{lcccccc}
\hline & \multicolumn{7}{c}{ Composição (\%) } \\
Ingredientes & UM & CZ & EE & PB & FB & CHO \\
\hline Farelo de Soja & 12,45 & 6,21 & 2,95 & 59,89 & 7,07 & 11,43 \\
Farelo de Milho & 15,13 & 1,26 & 2,58 & 7,23 & 15,11 & 58,69 \\
Farelo de Trigo & 13,83 & 4,8 & 2,46 & 19,11 & 10,07 & 49,73 \\
Torta de Cupuaçu (Figura 7) & 17,52 & 9,11 & 17,93 & 17,79 & 11,1 & 26,55 \\
Óleo de Soja & 0 & 0 & 100 & 0 & 0 & 0 \\
\hline${ }^{* *}$ Premix Vit. Min. & 0 & 100 & 0 & 0 & 0 & 0 \\
\hline
\end{tabular}

**P. Vitaminico e mineral/kg: fosforo 0,5\%; cobre 2,66mg; ferro $16,66 \mathrm{mg}$; iodo 0,25 mg; manganes $25 \mathrm{mg}$; zinco $16,6 \mathrm{mg}$; vit. A 3,33 UI; vit. E 2 UI; vit. C 1,000 ppm, vit. D3 800UI; vit B10,46mg; vit. B12 3,33mg; vit B2 1,66mg; vit K 0,52mg. L- lisina (99\%); Dl-metionina (99\%).

As rações foram formuladas de maneira a conterem $28 \%$ de $\mathrm{PB}$, estas fornecidas duas vezes ao dia (08:00 h e 16:00h), ao nível de 5\% da biomassa. A qualidade da água foi monitorada quinzenalmente, medindo-se os parâmetros de oxigênio dissolvido $(\mathrm{mg} / \mathrm{L})$, a temperatura $\left({ }^{\circ} \mathrm{C}\right), \mathrm{o}$ $\mathrm{pH}$, transparência $(\mathrm{cm})$, amônia $(\mathrm{mg} / \mathrm{L})$ e a alcalinidade $(\mathrm{mg} / \mathrm{L})$. Para isto foram utilizados oxímetros da marca YSI-55 e phmetro portátil Hanna-HI98103. Para as demais análises dos 
parâmetros físicos e químicos da água foi utilizado um kit colorimétrico.

\section{Avaliações do desempenho zootécnico}

A avaliação do desempenho dos juvenis de tambaqui submetidos a diferentes níveis de inclusão de torta que cupuaçu na ração foi realizado com base nos seguintes dados de desempenho:

Ganho de peso (GP) GP: peso final (g) - peso inicial $(\mathrm{g})$
Conversão alimentar aparente (CAA)

CAA: Quantidade de ração fornecida (g) / Ganho de Peso

\section{Sobrevivência (S\%)}

S\%: Total de peixes vivos ao final/ total de peixes início x 100 .

Ganho de peso diário $(\mathrm{g})$

GP: Peso final (g) - Peso inicial / Quantidade de dias.

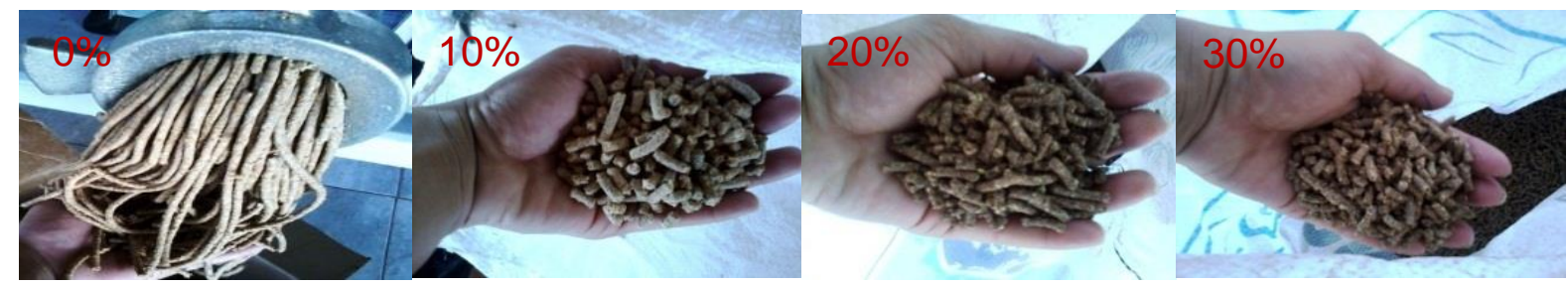

Figura 6. Dietas elaboradas contendo níveis crescentes de torta de cupuaçu $(0 \%, 10,20$ e $30 \%)$.

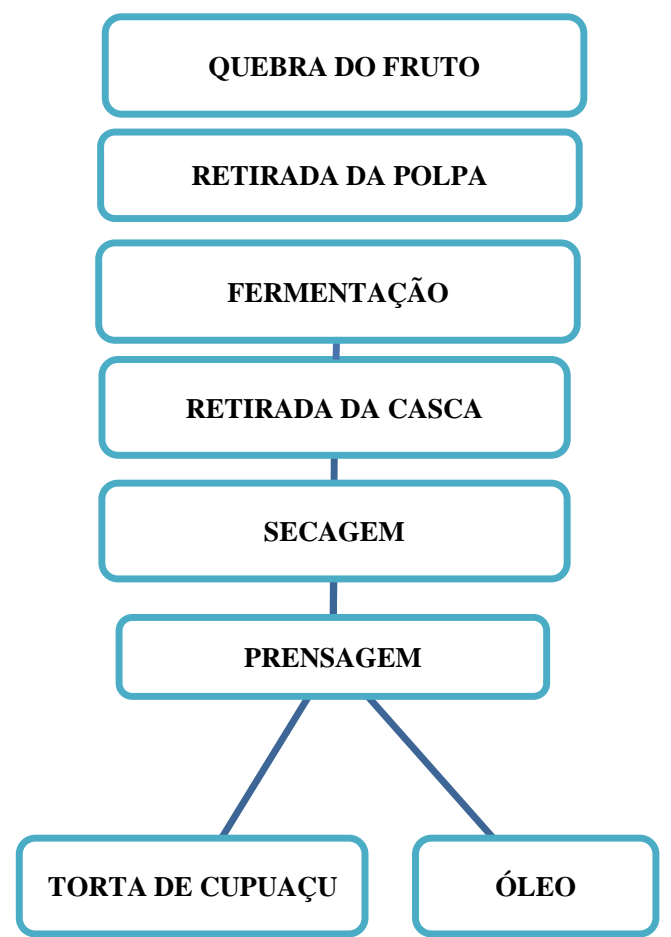

Figura 7. Preparação do ingrediente alternativo (torta de cupuaçu). Fonte: Carvalho et al. (2005).

Para a análise estatística, os dados foram submetidos a análise de variância ao nível de 5\% de probabilidade e em casos de significância foi utilizado o teste de Tukey ao nível de 5\% para discriminação das médias.

\section{Resultado e discussão}

Os parâmetros físicos e químicos da água permaneceram uniformes ao longo do experimento, não apresentando diferenças significativa entre os tratamentos $(\mathrm{p}<0,05)$, disposta na (Tabela 2).

Tabela 2. Valores médios dos parâmetros físico e químicos monitorados da qualidade de água da unidade experimental

\begin{tabular}{lc}
\hline Parâmetros & Valores médios \\
\hline *O.D (mg/l) & $4,7 \pm 2,1$ \\
Temperatura (oC) & $32,2 \pm 5,2$ \\
pH & $7,6 \pm 2,4$ \\
Transparência (cm) & $54 \pm 15$ \\
Amônia (mg/l) & $0,3 \pm 0,1$ \\
Alcalinidade (mg/l) & $4,0 \pm 1,0$ \\
\hline
\end{tabular}

*O.D- Oxigênio Dissolvido; Média \pm desvio padrão.

Os valores dos parâmetros medidos foram: Oxigênio Dissolvido (4,7 $\quad \pm \quad 2,1 \mathrm{mg} / \mathrm{l})$, Temperatura $\left(32,2 \pm 5,2^{\circ} \mathrm{C}\right), \mathrm{pH}(7,6 \pm 2,4)$, Transparência $(54 \pm 15 \mathrm{~cm})$, Amônia Total $(0,3 \pm$ $0,1 \mathrm{mg} / \mathrm{l})$, e Alcalinidade $\left(4,0 \pm 1,0 \mathrm{Ca} \mathrm{CO}_{3} / \mathrm{l}\right)$.

\section{Índices zootécnicos}

Durante os 60 dias de experimento, o índice de taxa sobrevivência foi de $100 \%$ durante todo o estudo em todos os tratamentos, indicando que as dietas elaboradas não geraram mortalidade aos juvenis. Não houve diferença significativa (p>0,05) entre os tratamentos de 10, 20, e 30\% de inclusão, em relação ao ganho de peso, conversão alimentar aparente e ganho de peso diário, 
diferenciando apenas do tratamento controle (Tabela 3).

Nesse trabalho, o ganho de peso diário (GDP) dos juvenis de tambaqui ficou em 1,16 g/dia para peixes alimentados com a dieta controle e de 0,95 ; 0,96 e 0,83 para os peixes alimentados com $10 \%$, $20 \%$ e $30 \%$ de inclusão do produto alternativo (torta de cupuaçu) respectivamente.

Os resultados encontrados de conversão alimentar aparente neste estudo foram inferiores aos descritos por Lima et al. (2009) com tambaqui criado em viveiros e alimentados com rações alternativos elaboradas com resíduos de abacaxi, onde foram obtidos ganhos de peso diário de 0,58 g e conversão alimentar aparente de 2,2:1. Torelli et al. (2010), ao trabalharem com resíduos agroindustriais na alimentação de tambaqui em sistema de policultivo obtiveram um ganho de peso médio diário de $0,19 \mathrm{~g}$ inferior ao descrito no presente estudo.

Tabela 3. Parâmetros de desempenho ( $\mathrm{S}$ - Sobrevivência; GP - Ganho de Peso; CAA - Conversão Alimentar Aparente; GDP - Ganho de peso diário)

\begin{tabular}{lcccc}
\hline Tratamento & $\mathrm{S}(\%)$ & $\mathrm{GP}(\mathrm{g})$ & $\mathrm{CAA}$ & $\mathrm{GPD}(\mathrm{g} /$ dia $)$ \\
\hline $0 \%$ de inclusão $\left(^{*}\right)$ & $100^{\mathrm{a}}$ & $69,63 \pm 18,8^{\mathrm{a}}$ & $2,60: 1^{\mathrm{a}}$ & $1,16^{\mathrm{a}}$ \\
$10 \%$ de inclusão $(*)$ & $100^{\mathrm{a}}$ & $56,9 \pm 17,8^{\mathrm{b}}$ & $3.16: 1^{\mathrm{b}}$ & $0,95^{\mathrm{b}}$ \\
$20 \%$ de inclusão $\left(^{*}\right)$ & $100^{\mathrm{a}}$ & $57,4 \pm 17,1^{\mathrm{b}}$ & $3.13: 1^{\mathrm{b}}$ & $0,96^{\mathrm{b}}$ \\
$30 \%$ de inclusão $\left(^{*}\right)$ & $100^{\mathrm{a}}$ & $50 \pm 15,4^{\mathrm{b}}$ & $3.57: 1^{\mathrm{b}}$ & $0,83^{\mathrm{b}}$ \\
\hline
\end{tabular}

"Letras diferentes na mesma coluna são diferentes (p<0,05) pelo teste de Tukey. (*) Níveis de inclusão de torta de cupuaçu S: Sobrevivência; GP: Ganho de peso; CAA: Conversão Alimentar Aparente; GPD: Ganho de peso Diário.

Os resultados encontrados de conversão alimentar aparente neste estudo foram inferiores aos descritos por Lima et al. (2009) com tambaqui criado em viveiros e alimentados com rações alternativos elaboradas com resíduos de abacaxi, onde foram obtidos ganhos de peso diário de 0,58 g e conversão alimentar aparente de 2,2:1. Torelli et al. (2010), ao trabalharem com resíduos agroindustriais na alimentação de tambaqui em sistema de policultivo obtiveram um ganho de peso médio diário de $0,19 \mathrm{~g}$ inferior ao descrito no presente estudo. Contudo, resultados semelhantes foram descritos por Lima et al. (2009) onde avaliaram o desempenho de tilápia do Nilo (Oreochomis niloticus) alimentado com diferentes níveis de inclusão de resíduos de abacaxi. Os resultados obtidos foram de um ganho de peso diário (GDP) de $0,71 \mathrm{~g}$ para a ração controle $(0 \%)$ e de $0,96 \mathrm{~g}$ para a ração com $10 \%$ de inclusão de abacaxi.

Os juvenis de tambaqui apresentaram uma conversão alimentar aparente satisfatórios. Onde a ração controle $(0 \%)$ foi a mais adequada entre os tratamentos, atribuindo-se uma conversão de 2.60:1, sendo diferente estatisticamente $(p>0,05)$ dos tratamentos com inclusão de torta de cupuaçu. As rações com adição do ingrediente alternativo não apresentaram diferenças significativas entre elas $(p<0,05)$. Quanto ao ganho de peso, foi evidenciado que os melhores resultados para este índice foram dos animais submetidos a alimentação controle (Figura 8).

Em relação ao ganho de peso, os resultados encontrados neste trabalho ratificam ao da pesquisa realizada por Lopes et al. (2010), onde foram avaliados níveis crescentes $(5,10,30 \%)$ e farelo de babaçu em dietas para tambaqui. Foi observada que a inclusão deste ingrediente alternativo não influenciou $(\mathrm{p}>0,05)$ sobre $\mathrm{o}$ desempenho produtivo dos juvenis de tambaqui entre os níveis testados. Segundo o autor as reservas de energia presentes no fígado seriam foram utilizadas para compensar alguma perda energética, ou mesmo em resposta à ação de algum fator antinutricional presente no ingrediente.

Lima et al. (2009) ao trabalharem com tilápia do Nilo (Oreochromis niloticus), alimentados com diferentes níveis de resíduos de abacaxi $(2,5,7$ e $10 \%)$ também não identificaram melhora ( $p>0,05)$, no desempenho produtivo da espécie estudada conforme o aumento dos teores do ingrediente alternativo. Segundo o NRC (2011) as rações compostas por ingredientes de origem vegetal devem conter de 3 a $5 \%$ de fibra bruta. A utilização de elevados teores de fibra na dieta causa decréscimo na taxa de esvaziamento gástrico, decorrente de menor ingestão e digestibilidade dos nutrientes (Hilton et al., 1983). 


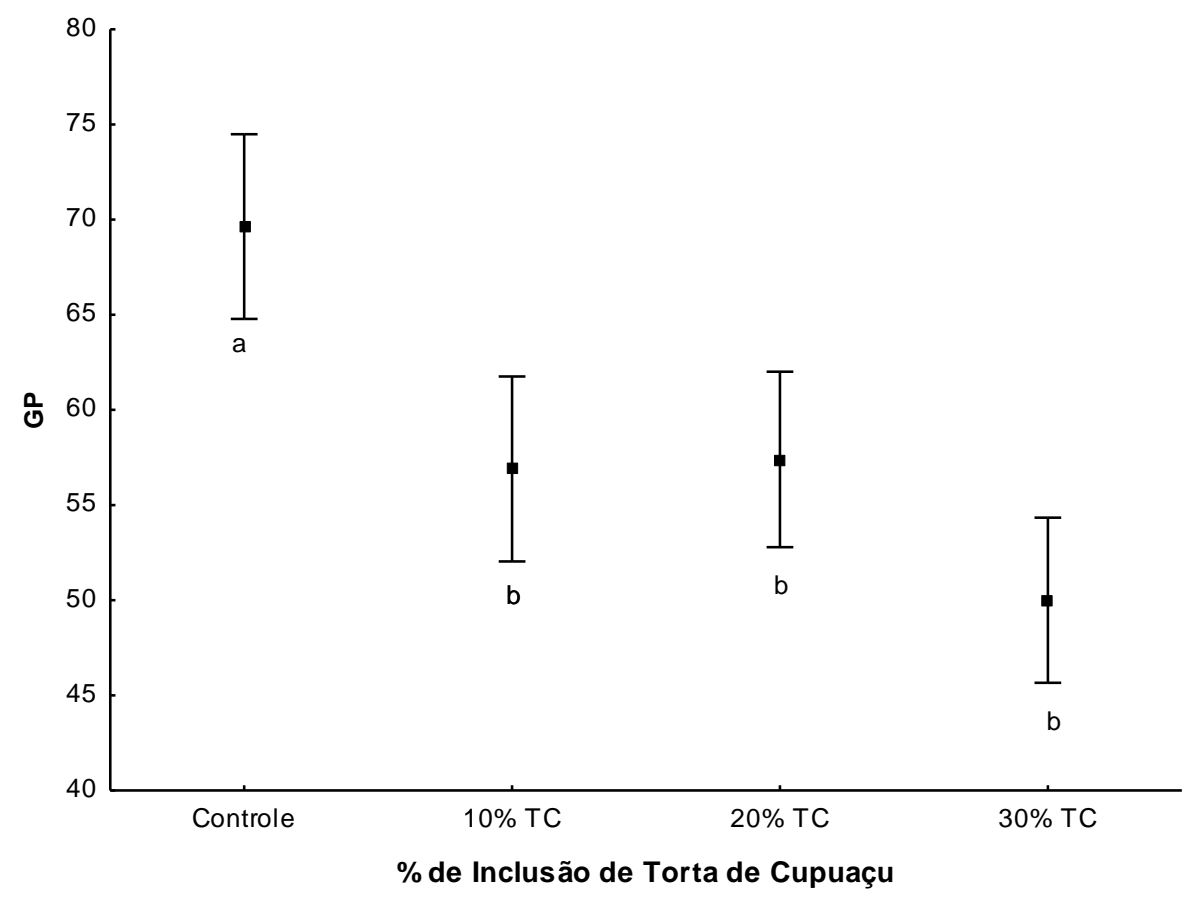

Figura 8. Relação de ganho de peso dos animais experimentais utilizados neste trabalho. Usados no teste para avaliar o desempenho produtivo de juvenis de tambaqui alimentados com rações peletizadas contendo níveis crescentes de torta de cupuaçu.

Os resultados deste trabalho corroboram o argumento acima, uma vez que quanto maior a inclusão da torta de cupuaçu na dieta para os juvenis, menor o desempenho corporal da espécie, indicando que o teor de fibra bruta encontrada no ingrediente alternativo intervém de forma negativa na digestibilidade dos nutrientes. Os resultados de desempenho zootécnico apresentado por (Hilton et al., 1983) foram semelhantes a esta pesquisa. Os autores realizaram um estudo com trutas, onde observaram que os teores de fibra bruta em níveis de $10 \%$ a $20 \%$ diminuem o coeficiente de digestibilidade dos nutrientes da ração.

A redução da digestibilidade da energia e do extrato etéreo nos maiores níveis de fibra pode ser atribuída ao fato da fibra agir na captação de micelas de gordura no intestino reduzindo a disponibilidade da energia (Madar and Thorne, 1987). Nos experimentos concretizados por Thebaudin et al. (1997) e Montagne et al. (2003), os níveis de fibra acima de $10 \%$ provocaram efeito negativo na digestibilidade, o que pode ser atribuído à característica da fibra insolúvel de reter maior quantidade de água no bolo alimentar dificultando a ação dos sais biliares e enzimas digestivas.

\section{Conclusão}

A inclusão de $10 \%, 20 \%$ e $30 \%$ de torta de cupuaçu na dieta para peixe, resultou em piora nas variáveis de desempenho zootécnicos dos juvenis de tambaqui. Isto pode estar diretamente relacionado com a elevada concentração de fibra bruta encontrada neste ingrediente alternativo.

\section{Referências bibliográficas}

Baldisserotto, B. (2004). Criação de jundiá. Universidade Federal de Santa Maria, Santa Maria.

Bezerra, S. K., Souza, R. C., Melo, J. F. B. \& Campeche, D. F. B. (2014). Crescimento de tambaqui alimentado com diferentes niveis de farinha de manga e proteína na ração. Archivos de Zootecnia, 63, 587-598.

Calzavara, B. B. G., Muller, C. H. \& Kahwage, O. N. C. (1984). Fruticultura tropical: o cupuaçuzeiro, cultivo, beneficiamento $e$ utilização do fruto. EMBRAPA-CPATU, Ilhéus.

Carvalho, A. V., Garcia, N. H. P. \& Wada, J. K. A. (2005). Caracterização físico-química e curvas de solubilidade protéica de sementes, amêndoas fermentadas e torradas de cupuaçu (Theobroma grandiflorum Schum). Brazilian Journal of Food Technology, 8, 127-134. 
Chagas, E. C., Carvalho, G. L., Júnior, H. M., Roubach, R. \& Paula Lourenço, J. N. (2005). Desempenho de tambaqui cultivado em tanques-rede, em lago de várzea, sob diferentes taxas de alimentação. Pesquisa Agropecuária Brasileira, 40, 833-835.

Claro-Jr, L., Ferreira, E., Zuanon, J. \& AraujoLima, C. (2004). O efeito da floresta alagada na alimentação de três espécies de peixes onívoros em lagos de várzea da Amazônia Central, Brasil. Acta Amazonica, 34, 133-137.

Dairiki, J. K. \& Silva, T. B. A. (2011). Revisão de literatura: exigências nutricionais do tambaqui-compilação de trabalhos, formulação de ração adequada e desafios futuros. In: EMBRAPA (ed.) Manaus: Embrapa Amazônia Ocidental. Manaus.

De-Carvalho, H. R. L., Souza, R. A. L. \& Cintra, I. H. A. (2013). A aquicultura na microrregião do Guamá, Estado do Pará, Amazônia Oriental, Brasil. Ciências Agrárias, 56, 1-6.

FAO. (2010). The state of world fisheries and aquaculture. FAO, 145.

FAO. (2015). Statistical Yearbook. Food and Agriculture Organization of the United Nations, Rome, Italy.

Hilton, J. W., Atkinson, J. L. \& Slinger, S. J. (1983). Effect of increased dietary fiber on the growth of rainbow trout (Salmo gairdneri). Canadian Journal of Fisheries and Aquatic Sciences, 40, 81-85.

Lima, M. R., Ludke, M. d. C. M. M., Neto, F. d. F. P., Holanda, M. C. R., Santos, E. L., Torres, T. R., Pinto, B. W. C., Lins, S. E. B. \& Souza, K. K. (2009). Desempenho de tilápia do Nilo (Oreochromis niloticus) alimentadas com diferentes níveis de resíduos de abacaxi. Recife.

Lopes, J. M., Pascoal, L. A. F., Silva Filho, F. P., Santos, I. B., Watanabe, P. H., Araujo, D. d. M., Pinto, D. C. \& Oliveira, P. S. (2010). Farelo de babaçu em dietas para tambaqui. Revista Brasileira de Saúde e Produção Animal, 11.

Madar, Z. \& Thorne, R. (1987). Dietary fiber. Progress in Food and Nutrition Science, 11, 153-174.

Melo, L. A. S., Izel, A. C. U. \& Rodrigues, F. M. (2001). Criação de tambaqui (Colossoma macropoтит) em viveiros de argila/barragens no Estado do Amazonas. In: EMBRAPA (ed.) Embrapa Amazônia Ocidental. Manaus.
Montagne, L., Pluske, J. R. \& Hampson, D. J. (2003). A review of interactions between dietary fibre and the intestinal mucosa, and their consequences on digestive health in young non-ruminant animals. Animal Feed Science and Technology, 108, 95-117.

NRC. (2011). Nutrient Requirements of Fish and Shrimp, 7th rev. edn. Natl. Acad. Press, Washington, DC., Washington.

Nwannaa, L., Oishi, C. \& Pereira-Filho, M. (2008). Use of phytase to improve the digestibility of alternative feed ingredients by Amazon tambaqui, Colossoma macropomum. Sci. Asia, 34, 353-360.

Pianesso, D., Lazzari, R., Mombach, P. I., Adorian, T. J., Uczay, J., Neto, J. R. \& Emanuelli, T. (2013). Substituição do farelo de soja pelo farelo de linhaça em dietas para a piava (Leporinus obtusidens). Semina: Ciências Agrárias, 34, 419-430.

Rotta, M. A. (2002). Use of energy and protein for fish. In: EMBRAPA (ed.) Embrapa: Pantanal. EMBRAPA, Corumba.

Sandoval Jr, P., Trombeta, T. D. \& Mattos, B. O. (2013). Manual de criação de peixes em taques-rede. EMBRAPA, Brasília.

Santos, C. A. A., Coelho, A. F. S. \& Carreiro, S. C. (2008). Avaliação microbiológica de polpas de frutas congeladas. Ciência e Tecnologia de Alimentos, 28, 913-915.

Sousa, M. S. B., Vieira, L. M., Silva, M. d. J. M. \& Lima, A. (2011). Caracterização nutricional e compostos antioxidantes em resíduos de polpas de frutas tropicais. Ciência Agrotecnologia, 35, 554-559.

Souza, A. G. C., Sousa, N. R., Silva, S. E. L., Nunes, C. D. M., Canto, A. C. \& Cruz, L. A. A. (1996). Fruteiras da Amazônia. EMBRAPACPAA, 1, 204.

Thebaudin, J. Y., Lefebvre, A. C., Harrington, M. \& Bourgeois, C. M. (1997). Dietary fibres: nutritional and technological interest. Trends in Food Science \& Technology, 8, 41-48.

Torelli, J. E., Oliveira, E. G., Hipolito, M. L. \& Ribeiro, L. L. (2010). Uso de resíduos agroindustriais na alimentação de peixes de policultivo. Revista Brasileira de Engenharia de Pesca, 5, 1-15.

Vieira, M. C., Teixeira, A. A. \& Silva, C. L. M. (2000). Mathematical modeling of the thermal degradation kinetics of vitamin $\mathrm{C}$ in cupuaçu 
(Theobroma grandiflorum) nectar. Journal of Food Engineering, 43, 1-7.

Villacorta-Correa, M. A. (1997). Estudo de idade e crescimento do tambaqui Colossoma macropomum (Characiformes: Characidae) no Amazonas Central, pela análise de marcas sazonais nas estruturas mineralizadas e microestruturas nos otólitos. In: Amazônia, I. N. d. P. d. (ed.). Instituto Nacional de Pesquisas da Amazônia, Manaus.

\section{Article History:}

Received 28 August 2016

Accepted 14 September 2016

Available on line 21 October 2016

License information: This is an open-access article distributed under the terms of the Creative Commons Attribution License 4.0, which permits unrestricted use, distribution, and reproduction in any medium, provided the original work is properly cited. 